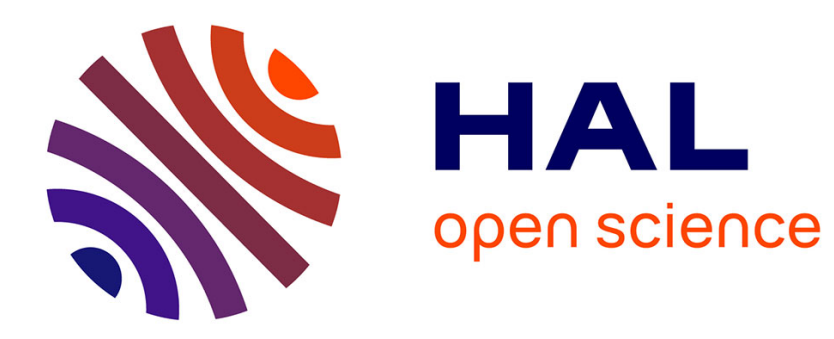

\title{
Paysages et paléoenvironnements quaternaires de la Provence aux Alpes occidentales
}

\author{
Vincent Ollivier
}

\section{To cite this version:}

Vincent Ollivier. Paysages et paléoenvironnements quaternaires de la Provence aux Alpes occidentales.

Quaternaire, 2014. hal-01766596

\section{HAL Id: hal-01766596 \\ https://hal.science/hal-01766596}

Submitted on 25 May 2018

HAL is a multi-disciplinary open access archive for the deposit and dissemination of scientific research documents, whether they are published or not. The documents may come from teaching and research institutions in France or abroad, or from public or private research centers.
L'archive ouverte pluridisciplinaire HAL, est destinée au dépôt et à la diffusion de documents scientifiques de niveau recherche, publiés ou non, émanant des établissements d'enseignement et de recherche français ou étrangers, des laboratoires publics ou privés. 


\section{QUAATERNAIRE}

Préface..

Michel DUBAR : Corrélation des gisements de mammifères villafranchiens de la région de (paléomon (bassin de Valensole, Apes de Haute-Provence, France) avec les autres sites dat Correlation of the Villafranchian mammalian sites of Puimoisson (Valensole basin, Alpes de Haute-Provence,
France) and others dated sites (palaeomagnetism, radionuclides) of Southern Europe (France, Italy and Spain) Vincent OLLIVIER, Frédéric MAGNIN, Jean Louis GUENDON \& Cécile MIRAMONT : Regard sur les dynamiques paysagères du Pléistocine supérieur (SIM-3 et SIM-2) du Luberon et
basse Provence (France) Perspectives on the Upper Pleistocene (MIS 3 and MIS 2) landscape dynamics of Luberon and Lower Provence
(Southern France)

Frédéric MAGNIN : Middle Pleniglacial to Holocene molluscan assemblages in the Marseille-

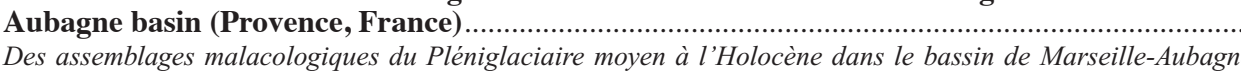
Des assemblages malacologiques du Pléniglaciaire moyen à l'Holocène dans le bassin de Marseille-Aubagne
(Provence, France) Cyril MONTOYA, Jean-Louis GUENDON, Jean-Baptiste BOUDIAS, Laurence BOUQUET, Mirco
DE STEFANI, Magali FABRE, Marie LAROCHE, Pierre LOPINET, Nicolas PORTALIER \&
Maryline RILLARDON : Le site paléolithique des Vaugreniers (Le Muy, Var) : un nouveau

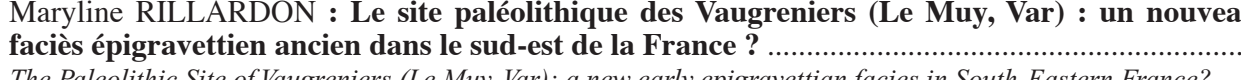
Elodie BRISSET, Cécile MIRAMONT, Frédéric GUITER, Fabien ARNAUD, Edward ANTHONY Domnés

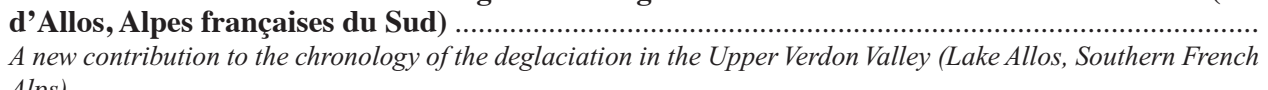

Adam A. ALI, Jean-Frédéric TERRAL, Vincent GIRARD \& Paul ROIRON : Les travertins à empreintes, témoins de la paléobiodiversité végétale ...........
Plant imprints from travertines, witnesses of plant palaeobiodiversity Frédéric MAGNIN \& Stéphane BONNET : Une succession malacologique du Pléniglaciaire
moyen et du Postglaciaire à Aix-en-Provence (France) : éléments de datation, taphonomie des assemblages et paléoenvironnements ...................................................................................
A Middle Pleniglacial and Postglacial molluscan succession at Aix-en-Provence (France): chronological data,
assemblages taphonomy and palaeoenvironments

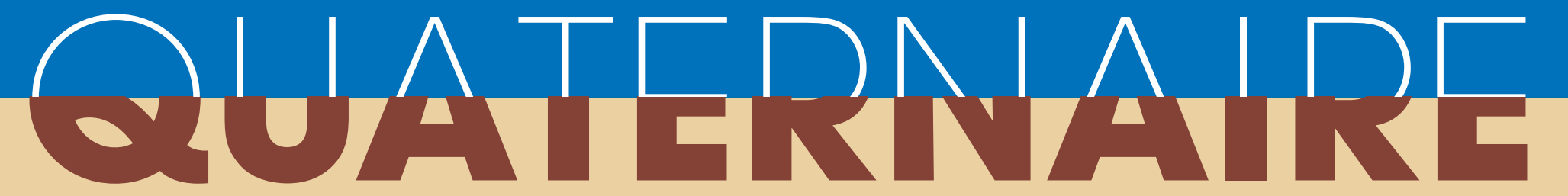

Revue de l'Association Française pour l'Étude du Quaternaire - CNF INQUA International Journal of the French Quaternary Association - CNF INQUA
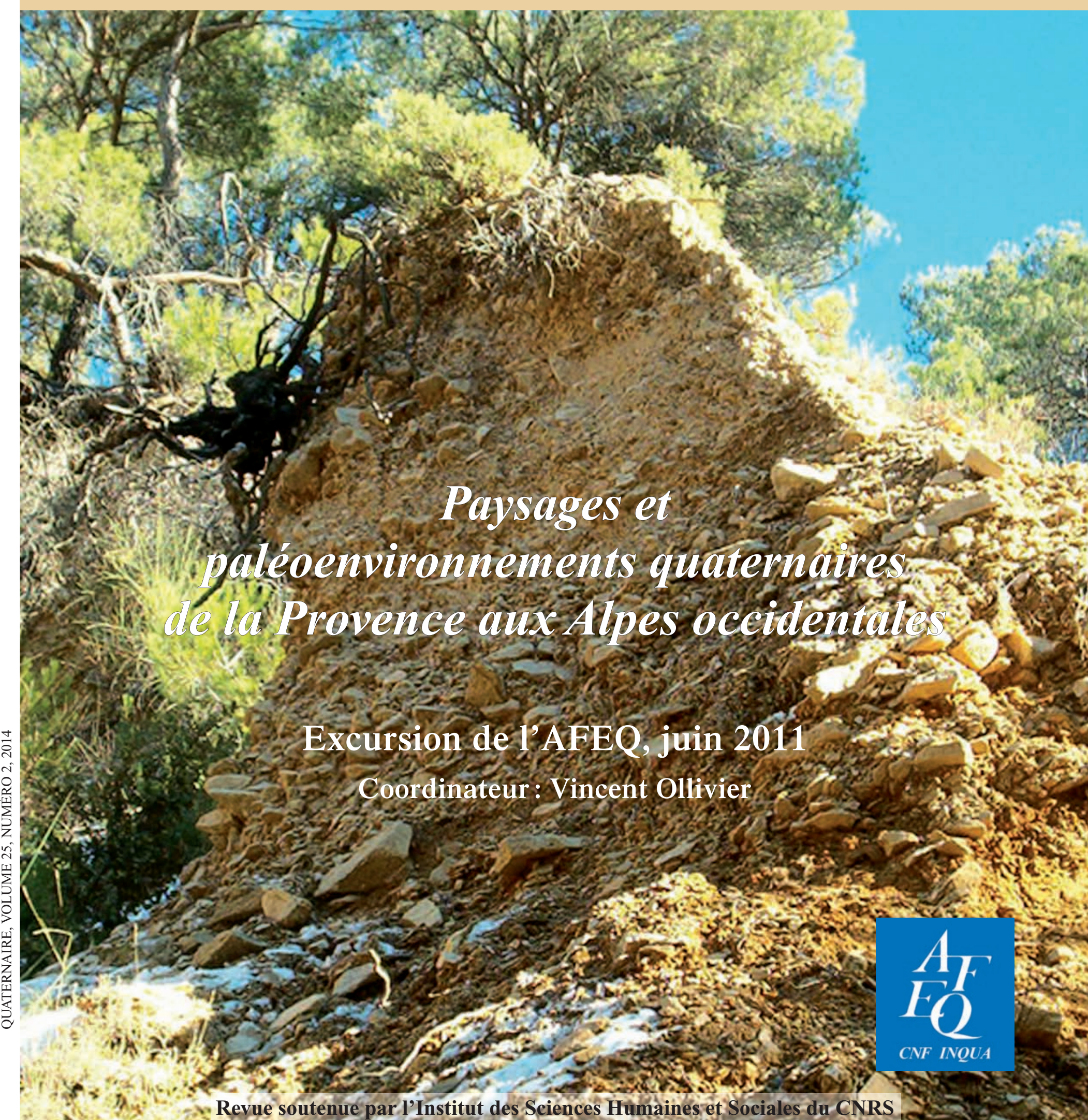
16 3 Revue soutenue par I'Institut des Sciences Humaineset Sociales du CNRS
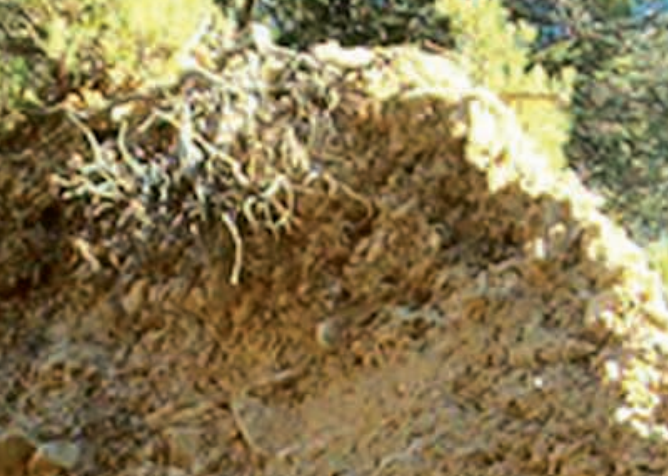

ra.

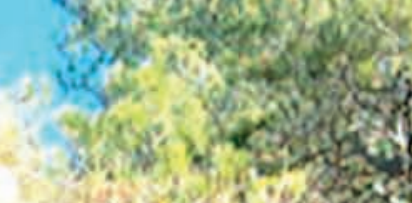

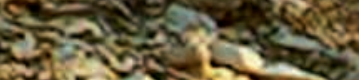




\section{QUATERNAIRE}

Conseil de l'Association et Comité de Rédaction / Association's Board and Drafting Committee P. ANTOINE, J.-J. BAHAIN, S. BALESCU, F. BASSINOT, P. BERTRAN, M.-A. COURTY, S. COUTARD, E. DEFIVE,

\section{M.F. SANCHEZ-GONI, A.-V. WALTER-SIMONNET}

\section{Bureau / Bureau}

Président / President : Jean-Jacques BAHAIN, Département de Préhistoire du Muséum national d'Histoire naturelle, Institut de

Vice-Président / Vice-president : Franck BASSINOT, Laboratoire des Sciences du Climat et de l'Environnement, avenue de la Terrasse, F-91198 Gif-sur-Yvette cedex (franck.bassinot@lsse.ipsl.frr).

Secrêtaire / Secretary : Sylvie COUTARD, INRAP Nord-Picardie, 518, rue Saint-Fuscien, F-80000 Amiens (sylvie.coutard@inrap.fr), Trésorier / Treasurer : Sanda BALESCU, Université Lille 1, Laboratoire Halma-Ipel, UMR 8164, Bâtiment de Géographie, F-5965

Directeur des relations internationales / Director of international relations : Maria Fernanda SÁNCHEZ-GOÑI, EPHE Directeur de la publication / Publicutean 1, avector : Pascace RUFFALDI L

\section{Adjoints à la Publication / Assistants of Publication}

Adjoint au Directeur de la Publication / Assistant of Publication director : Anne-Véronique WALTER-SIMONNET Laboratoire Chrono-Environnement, 16, route de Gray, F-25030 Besançon cedex (anne-veronique.walter@univ-fcomte.fr). Secrétariat d'édition / Edition secretary :

Olivier MOINE, Laboratoire de Géographie Physique, 1 place A. Briand, F-92195 Meudon cedex (oliviermoine@enrs-bellevue.fr). Mari-Thé

\section{Comité éditorial / Editorial committee}

\begin{tabular}{|c|c|c|c|}
\hline 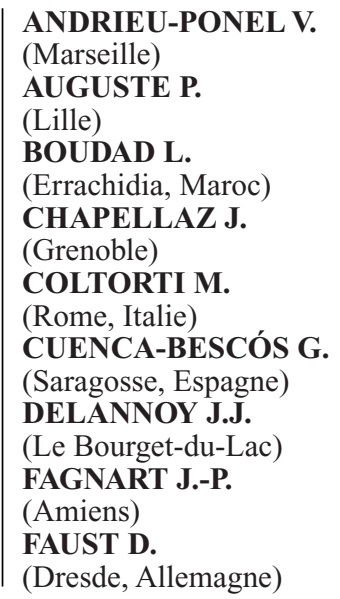 & 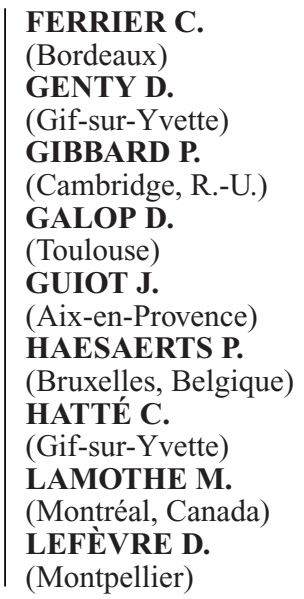 & 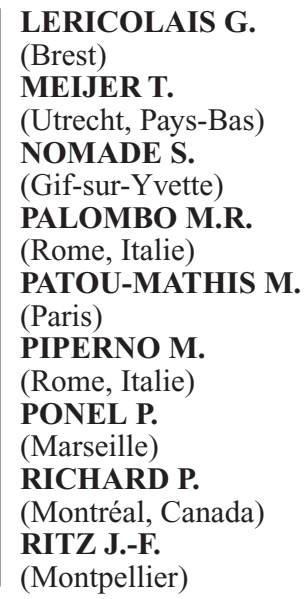 & $\begin{array}{l}\text { SCHLÜCHTER C. } \\
\text { (Berne, Suisse) } \\
\text { SCHREVE D.C. } \\
\text { (Londres, R.-U.) } \\
\text { THOORET J.-C. } \\
\text { (Clermont-Ferrand) } \\
\text { VAN KOLFSCHOTEN T. } \\
\text { (Leiden, Pays-Bas) } \\
\text { VANDENBERGHE J. } \\
\text { (Amsterdam, Pays-Bas) } \\
\text { (AELLACC. } \\
\text { (Aix-en-Provence) } \\
\text { vERECCHHA E. } \\
\text { (Lausanne, Suisse) } \\
\text { WILLIAMSON D. } \\
\text { (Aix-en-Provence) } \\
\text { ZÖLLER L. } \\
\text { (Bayreuth, Allemagne) }\end{array}$ \\
\hline
\end{tabular}

La correspondance relative à la publication est à adresser au Directeur de la publication, les règlements et les commandes au Trésorier, et toute autre correspondance au Secrétaire / Correspondence concerning pubce
the Treasurer, and any other correspondence to the Secretary.

Adhésion à l'AFEQ \& Abonnement à Quaternaire / AFEQ membership \& Quaternaire subscription

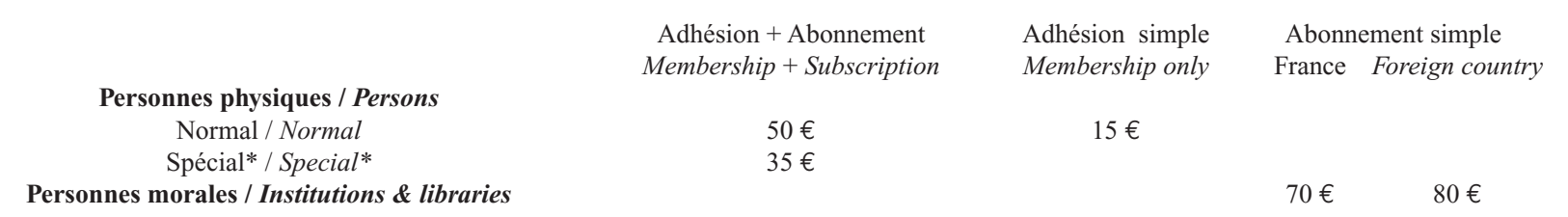

Prix TTC. Paiements par carte bancaire ou chèque à l'ordre de l'AFEQ / Prices including taxes. Payments by credit card or bank transfer forreign Tarif spécial étudiants, hors statuts et seniors (avec photocopie de la carte d'étudiant en cours de validité) / Special price for students, non-academic staff and seniors (with the copy of a valid student card).

Plus d'information sur le site internet/More information on the website: http://www.afeq.cnrs-bellevue.fr Photo de couverture / Cover picture : Substrat miocène à perforations de lithophages (premier plan) dans le massif du en vallons plio-pléistocènes une soirée d'hiver... / Traduction légende. (Photo : Ollivier V., 2014, la Motte-d'Aigues).

Conception et réalisation de la couverture / Coverage design and implementation : André Zetlaoui
RÈGLES DE PUBLICATION

QUATERNAIRE est une revue internationale, publiée sous l'egide "AFEQ. Elle accepte des articles traitant de tous les aspects de l'étude Interdisciplinaire. Les opinions émises n'engagent que la responsabilitré des signataires. Les articles sont obligatoirement soumis au Comité $d e$ lecture qui consulte deux specialistes du theme tratté. Des modifif

QUATERNAIRE publie des articles brefs (4-6 pages maximum, llustrations comprises) et des articles longs (15-20 pages maximum, llustrations comprisess ainsi que des analyses d'ourrages. Une page 6000 signes et intervalles. Les dépassements sont tolérés, mais leur coût d'impression est alors entièrement à la charge des auteurs. Les articles bresfs bénéficient d'un déla d'impression court (le premier numéro à p

Texte - QUATERNAIRE est publié en français ou en anglais. $\underline{\text { Le }}$ Itre, le résumé, les mots-clés et les légendes des illustrations doivent être présenté sur un format $21 \times 297 \mathrm{~cm}$ à double interligne avec une marge gauche de $3 \mathrm{~cm}$ et ordonné comme suit: titre (court, mais explicite en français et anglais); prenoms et noms des auteurs, adresses professionnelles et e-mail; résumé et abstract conséquents; mots-clés
(en francais et anglais); texte principal ; bibliographie; titres et el légendes 列 anglais. La revue n'accepte pas les notes de bas de page.

Illustrations - Les illustrations doivent être réductibles à une taille maximum de $17 \times 24 \mathrm{~cm}$ et ne doivent en aucun cas dépasser le format
A4. Il est recommandé d'adapter la taille des figures soit à la largeur otale du format de la revue $(17 \mathrm{~cm})$ soit à la largeur d'une colonne $8 \mathrm{~cm}$ ). Les originaux des figures dessinées par ordinateur, de préférence
déjà réduits à la taille d'impression finale, devront être imprimés sur un déjà réduits à la taille d'impression finale, devront être imprimés sur un
papier de qualité supérieure à une résolution élevée ( $2600 \mathrm{DPI}$ ) et ournis lors du premier envoi du manuscri.

Les photographies bien contrastées doivent être regroupées sur une même planche et comporter les échelles. Les originaux ou une copie de Les photographies et figures en collowrs sont acceptées mis lewr coût d'impression est à la charge des auteurs. Toutes les illustrations (figures, tableaux et planches) seront numérotées en chiffreses arabes. pans le cas où des lilustrations provenant dé documents dejà préalable l'autorisation de reproduction auprès des ayants-droits. Une copie de l'autorisation doit être jointe à l'envoi du manuscrit.

Références bibliographiques - La liste des références comportera des figures. Elles doivent être présentées par ordre alphabétique, puis chronologique (sans tenir compte des auteurs multiples). Les noms des périodiques seront donnés in extenso. Se conformer à la présentation

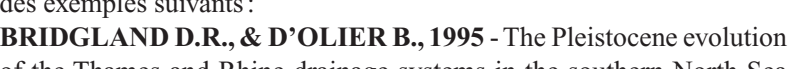
of the Thames and Rhine drainage systems in the southern North Sea
basin. In R.C. Preece (ed.), Island Britain: a Quaternary perspective. basin. In R.C. Preece (ed.), Island Britain: a Quaternary perspective.
Geological Society Special Publication, London, $96,27-45$. Geological Society Special Publication, London, $96,27-45$.
GIRET A., 1995 - Etude des déformations quaternaires du Roussillon. Quaternaire, 6 (3-4), 121-137. PUISSÉGUR J.J., 1976 - Mollusques continentaux quaternaires de
Bourgogne. Mémoires Géologiques de l'Université de Dijon, 3, Doin,
Paris 241 p.

envoyé en 1 exemplaire papier (texte et figures) ET sous forme d'un fichier PDF (texte et figures) au Directeur de la publicatio

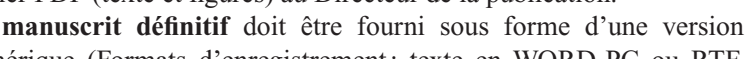
ableaux en Excel PC, illustrations (en 600dpi) en Adobe-Illustrator ou Adobe-Photoshop ou EPS, PICT, TIFF, sur CD Rom). La version
du logiciel utilisé et le format des fichiers doivent être spécifiés sur e support (CD Rom). Les premières êpreuves sont envoyées pour

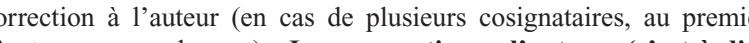

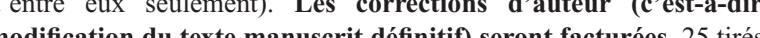
part seront fournis gratuitement ainsi qu'un PDF.

INSTRUCTIONS TO AUTHORS

QUATERNAIRE is an international journal published by the AFEQ. Papers on all aspects of Quaternary research are acceptec QUITERNIRE represent the op re submitted to the editor comminttee and sent to two reviewers Modifications might be asked to the author

Short papers should not exceed 4-6 printed pages (including figures) find long papers should not exceed $15-20$ printed pages (including
figue printed page (size $21 \times 29.7 \mathrm{~cm}$ ) corresponds broally to 6000 letters and spaces. The authors will be charged for exceeding pages (pages, pictures and tables). Short papers are published quickly 列

Text - QUATERNAIRE accepts papers in French or English. Titte 列,

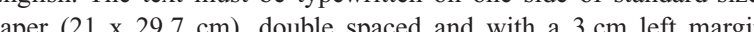
but brief, in French and in English); author's name; professional addres and e mail; abstract in English and in French ("résumé"); key-words in

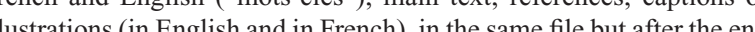
of the paper. Footnotes are not accepted.

Illustrations - Illustrations should be reducible to a maximum size of entire width of the type area $(17 \mathrm{~cm}$ ) or for the column width of $8 \mathrm{~cm}$ High-quality prints of figures (at least $600 \mathrm{DPI}$ ), preferably reduced to the final size, must be submitted with the manuscript. Photograph are to be sharply contrasted, grouped on the same plate and scaled
Originals or high-quality copies must be sent with the Colour photographs and illustrations are permitted provided the author
bear the cost. All illustrations (figures, tables and plates) should be numbered in Arab numerals

used, the author must have copt the permission of publication directly with the rightfful owner
Cormission must be send with the manuscript.

References-All publications cited in the text, the figures and the caption hould be listed. References should be presented in alphabetical an BRIDGLAND D.R., \& D'OLIER B., 1995 - The Pleistocene evolution of the Thames and Rhine drainage systems in the southern North Sea
basin. In R.C. Preece (ed.), Island Britain: a Quaternary perspective. GIRET A., 1995 - Etude des déformations quaternaires du Roussillon Quaternaire, 6 (3-4), 121-137.

PUISSÉGUR J.J., 1976 - Mollusques continentaux quaternaires Bourgogne. Mémoires Géologiques de l'Université de Dijon, 3, Doin,
Paris, 241 p.

Modalities of acceptance and printing - A PDF file (text and illustrations) AND one copy (text and illustrations) of the manuscrip
have to be sent to the Editor (After revision athors will send oue high quality printed copy of their paper (i.e. text and illustrations in $600 \mathrm{DP}$ ) and a disk: text in WORD for PC or RTF, tables in EXCEL for PC illustrations in Adobe-Illustrator, Adobe-Photoshop or EPS, TIFF, PICT
The following media can be used. flopyy disk formatted for PC, 100M Zip disk, CD ROM or DVD ROM Soffware and fles formats must be specified on the media. Proofs will be sent to the first author. The author will be charged for changes against the manuscript made by him in the 


\title{
PAYSAGES ET PALÉOENVIRONNEMENTS QUATERNAIRES DE LA PROVENCE AUX ALPES OCCIDENTALES
}

\author{
Vincent Ollivier ${ }^{1,2}$
}

Il n'est pas évident de pouvoir parcourir de manière exhaustive le champ des recherches sur le Quaternaire d'un aussi bel et vaste espace géographique que celui de la Provence et des Alpes occidentales. A l'instar des paysages de cette région, ils y sont d'une richesse et d'une complexité rares. Il est également difficile d'énoncer l'ensemble des données fondatrices sur lesquelles reposent nos actuels travaux. Nous pouvons cependant saluer le caractère essentiel des nombreuses analyses paysagères lato sensu nous ayant directement inspiré et qui ont également orienté les travaux d'une quantité remarquable de géographes, géologues et préhistoriens spécialistes du Quaternaire du sud-est de la France au cours de ces dernières décennies. Un bel hommage, certainement encore trop incomplet, doit donc être réservé notamment à Mesdames et Messieurs P. Ambert, J.-J. Blanc, M.-F. \& E. Bonifay, F. Bourdier, G. Clauzon, J.-L. de Beaulieu, A. Douguedroit, M. Dubar, J. \& P. Gabert, C. Gouvernet, J.-L. Guendon, G. Guieu, M. Jorda, M. Julian, G. Montjuvent, R. Neboit-Guilhot, J. Nicod, M. Provansal, P. Roiron, C. Rousset, J. Riser, J.-J. Tiercelin, J.-M. Triat, J. Tricart, J. Vaudour et J.-L. Vernet.

La morphogenèse entre Provence et Alpes occidentales repose sur un climat spécifique et une généreuse orographie, dont les grands volumes montagneux résultent d'une efficace tectonique active à l'échelle des derniers millions d'années. Partout, l'érosion s'y exprime avec vigueur sous le jeu d'un rythme saisonnier contrasté. Une mosaïque de processus d'ablation peut être observée. Que ceux-ci soient liés aux périodes froides ou aux épisodes plus cléments, au cours de 1'année ou des millénaires. Transports et dépôts sédimentaires répondent également à cette règle de la variabilité, dictée par la diversité des contextes environnementaux sur un territoire restreint. Le géomorphologue, ou tout quaternariste, y est au paradis...

Dans ce volume spécial de la revue Quaternaire, nous avons voulu revisiter, ouvrir et compléter ce que nous avions présenté lors de l'excursion annuelle de l'AFEQ
(21-23 juin 2011) intitulée : " Paysages, travertins et paléoenvironnements quaternaires entre Provence et Alpes occidentales $"$. A cette occasion, des questions et découvertes portant sur l'évolution du domaine provençal et alpin depuis le Néogène jusqu'à l'extrême fin du Quaternaire avaient été exposées (travertinisations, pédogenèses, biostratigraphies, etc.). Ces thématiques sont ici reprises et agrémentées de nouveaux sujets de recherche.

Les articles publiés dans ce numéro concernent une large période chronologique et un important gradient bioclimatique (étagement altitudinal allant du méditerranéen à l'alpin, voir carte de localisation) sur des domaines privilégiés relevant de la géomorphologie, de la paléontologie, de la paléoécologie et de l'archéologie. Dans le secteur de Puimoisson (Alpes-deHaute-Provence), des corrélations biostratigraphiques et géochronologiques à l'échelle de l'Europe du Sud sont proposées pour la transition Pliocène-Pléistocène. Les derniers résultats chronostratigraphiques issus des formations de glacis d'accumulation à horizons pédogénétiques du Luberon (Vaucluse), fournissent l'occasion de présenter ici de nouvelles données et une synthèse sur les rythmes morphoclimatiques et mutations paysagères associées au cours des stades isotopiques marins 3 et 2, à 1'échelle des Alpes du Sud et de la Provence occidentale. Les analyses malacologiques réalisées dans le secteur de Gémenos (Bouches-du-Rhône) sur une séquence sédimentaire du Pléistocène supérieur et de l'Holocène, s'inscrivent d'ailleurs dans la continuité de ce type de recherche. Au niveau des sociétés méditerranéennes tardiglaciaires, c'est à partir des découvertes du site des Vaugreniers, localisé sur une ancienne terrasse alluviale de la Naturby (Le Muy, Var), que les connaissances au sujet de deux phases mal documentées de la tradition culturelle épigravettienne sont enrichies pour la Provence orientale. Dans la vallée du Haut Verdon, les datations ${ }^{14} \mathrm{C}$ AMS obtenues dans la séquence sédimentaire du lac d'Allos (Alpes-de-Haute-Provence), défini comme le plus grand lac naturel d'altitude en Europe, permettent de proposer une nouvelle chronologie de la

\footnotetext{
'Collège de France, Institut du Proche-Orient Ancien, CNRS, PrOCauLAC UMR 7192, FR-75005 PARIS.

Coutriel : vincent.ollivier@ollege-de-france.fr

${ }^{2}$ Aix-Marseille Université, CNRS, Ministère de la Culture et de la Communication, LAMPEA UMR 7269, FR-13094 AIX-EN-PROVENCE.

Courviel : ollivier@mmsh.univ-aix.fr
} 
déglaciation de ce secteur. Les formations travertineuses sont abordées selon une approche paléobotanique par l'intermédiaire des empreintes foliaires qu'elles peuvent contenir, précieux témoignages sur l'histoire de certaines espèces ligneuses et des paléoenvironnements du Pleistocène moyen à l'Holocène dans le sud de la France. Enfin, des analyses malacologiques et géomorphologiques, réalisées dans les accumulations sédimentaires alluviopalustres et archéologiques du site de Mignet à Aix-enProvence (Bouches-du-Rhône), révèlent une nouvelle et rare formation provençale datée du stade isotopique marin 3. Des intrusions de coquilles holocènes et la dernière partie historique de la séquence permettent en outre de discuter de l'importance du renouvellement des peuplements malacologiques qui s'est opéré dans le Miđi de la France en relation avec l'anthropisation.

Nous espérons ici avoir pu dresser un premier portrait suffisamment fourni de l'évolution des paysages quaternaires de Provence et des Alpes du Sud, en privilégiant les approches, la diversité des compétences et les échanges.

Je tiens particulièrement à remercier l'ensemble des auteurs et relecteurs de ce volume pour leurs contributions. Comme pour le legs de nos prédécesseurs, puissent elles modestement ouvrir la voie d'une certaine continuité et d'un renouveau dans les recherches entreprises sur le Quaternaire provençal et alpin.

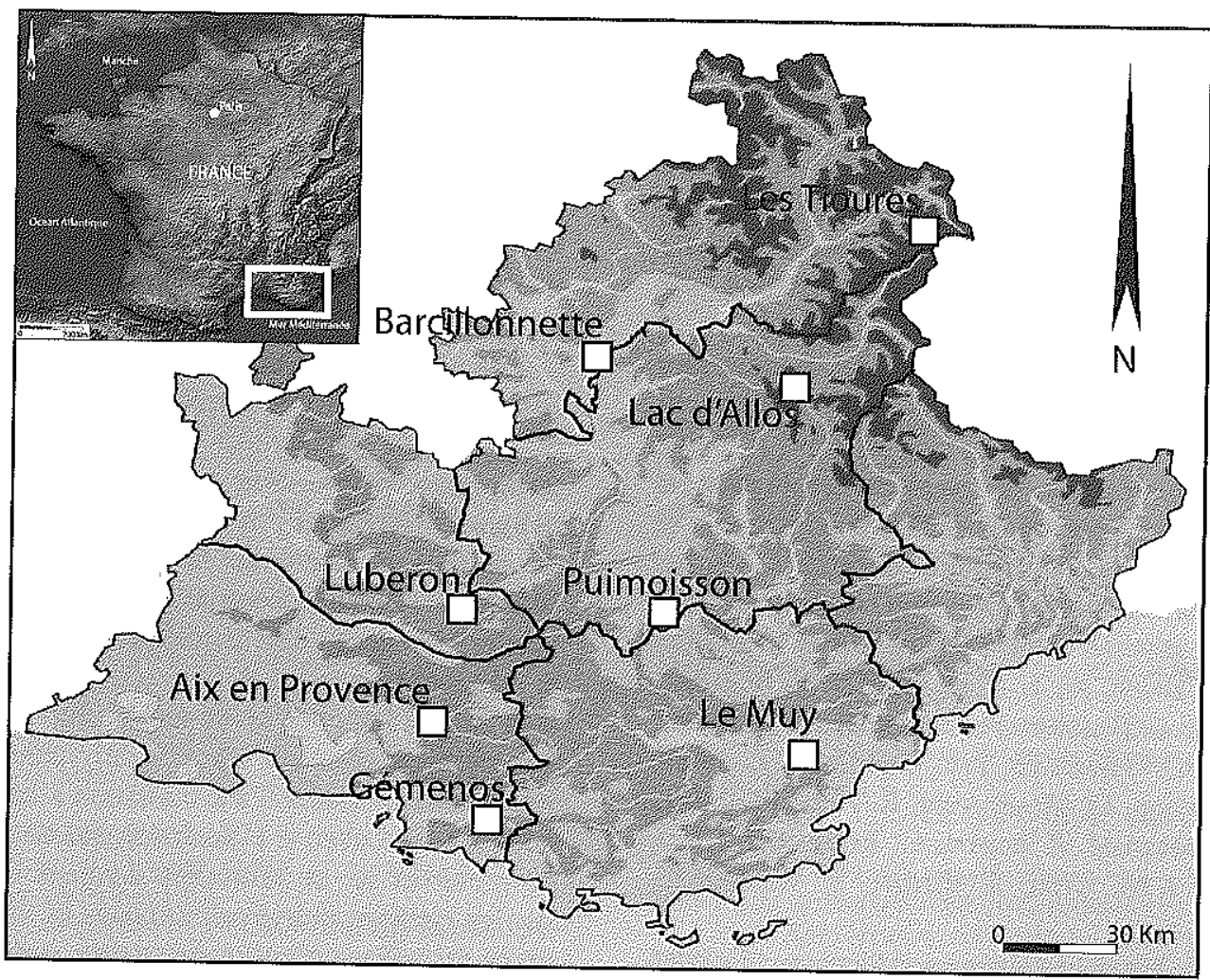

Répartition géographique des sites abordés dans ce volume par l'ensemble des auteurs 